\title{
Personalien und Tagesnachrichten
}

Dr. Wilh. Richter, Leiter der Hautabteilung der chii· urgischen Poli-klinik (Geh.-Rat Prof. Dr. Bier) in Berlin, hat sich fur Dermatologie habilitiert.

Prof. Dr. Rule, Direktor der Univ.-Hautklinik in Leipzig, wurde zum Ehrenmitglied der Griechischen Dermatologischen Gesellschaft gewählt, ebenso Prof. Gl·ans, Direktor der Frankfurter Hautklinik.

Dr. Clement Simon in Paris wurde zum Ehrenmitglied, Dr. Werner Jadassohn in Zurich, Prof. Kumer in Innsbruck, Dr. Alfred Perutz in Wien, Dr. Ernst Schulmann in Paris, Dr. Werner Worms in Berlin-Dahlem wurden zu korrespondierenden Mitgliedern der Società Italiana di Dermatologia e Sífilografia gewählt.

Generaloberarzt a. D. Dr. Arno Thalmaim in Dresden ist gestorben. Er war méhrere Jahre al·s Assistent an die Lessersche Klinik in Berlin kommandiert $u$,nd hat sich durch bakteriologische Untersuchungen besonders über Gonokokken auíSgezeichneti.

Von Prau Dohi in Tokio ist beim Hinscheiden ihres Mamies, des Prof. Keizo Dohi, der Berliner Dermatologischen Gesellschaft ein nennenswerter Geldbetrag geschenkt worden, der voraussichtlich für eine Dohi-Stiftung den Namen des Porschers in Erinnerung halten soil. Am 1. Juli 1932 sind 50 Jahre vergangen, seitdem die Hautklinik der Bonner Universität von Geh.-Rat Prof. J. Doutrelepont begründet wurde.

Auf der gemeinsamen Tagung der niederländischen und rheinisch- $\lambda$ vest-fälischen Dermatologen wurde am 16. April im Haag eine Reihe von Vor-trägen gehaiten; am 17. April wurde die Tagung in der Univ.-Hautklinik in Leiden abgehalt en, wo neben Demonstrationen und Krankenvorstellungen weitere Vorträge stattfanden.

In Dresden fand vom 3. bis õ. April 1932 die Tagung der Deutschen Röntgengesellsehaft statt, auf der außer physikalischen Problemen (Struktur-diagnose) auch dermatologische Pragen auf Grund von Referaten der Pro-fessoren Rost und Schreus besprochen wurden. 